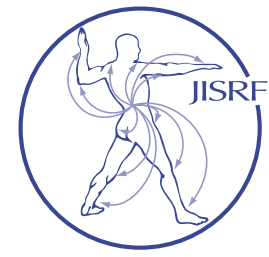

Check for updates

\title{
The 16-Year Evolution of Proximal Modular Stem Design - Eliminating Failure of Modular Junction
}

\author{
Tkach $T^{1}$, McTighe $T^{2}$
}

\section{Abstract}

Background: The complexity of hip reconstruction has been and continues to be a perplexing problem with restoring leg length, femoral offset, joint stability and overall hip implant fixation. These were contributing factors that lead to the development of a novel proximal femoral component design "Apex Modular Stem" (Omni, Raynham, MA). The basic stem geometry features a straight stem with a metaphyseal fit and fill cone, a medial triangle and a modular neck junction that allows for version and offset adjustment.

In recent years, there has been great concern with the use of modularity in total hip arthroplasty. The goals of this study are (1) to identify complications with the use of a proximal modular design and (2) demonstrated factors that have eliminated those complications.

Methods: This is a retrospective study of a single surgeon series (Generation I and Generation II) of using the same cementless stem and proximal modular neck body (Apex Modular Stem and Omni Mod Hip Stem) from 2000 to 2016 totaling 2,125 stems (483 Generation I and 1,642 Generation II).

Results: Generation I, 483 stems were implanted between 2000 and 2004 of which 31 alignment pins sheared resulting in a revision rate of $6.4 \%$. Generation II, 1,642 stems have been implanted between 2004 and 2016 all by the same surgeon with no failures of the modular junction.

Conclusion: All implant devices entail a multitude of risks and benefits. The Apex Modular Stem (Generation I), provided excellent fixation, minimal risk of modular junc- tion corrosion, and simple control of anteversion and femoral offset. The limitation was found to be the risk of the alignment pin shearing $(6.4 \%)$. The pin was enlarged to make it $225 \%$ stronger in torsional resistance, and in a subsequent series of over 1,600 femoral stems in a single surgeon series, there were no pin failures over a 12 year duration.

\section{Background}

Generation I (Apex Modular Stems) 483 were implanted between 2000 and 2004 on consecutive patients by the senior author. All were performed using the posterior approach. All cases used a mixture of different cementless acetabular components from a variety of manufactures.

Surgical stem and cup preparation and instrumentation has not changed between the two-generation stem designs.

The Dual Press modular junction employs two areas of cylindrical press-fit. To create this mechanical lock, the proximal and distal diameters of the peg are slightly larger than the corresponding holes in the stem, creating two bands of interference, or press-fit. Figure 1.

The proximal end of each stem includes an alignment pin that engages with a mating hole on the distal surface of each neck. This pin allows for anteversion position and for de-rotation of the modular neck on the stem. Figure 2.

Keywords: cementless stem; proximal modularity; modular junction

Level of Evidence: AAOS Therapeutic Level II

Educational Value \& Significance: JISRF Level B 


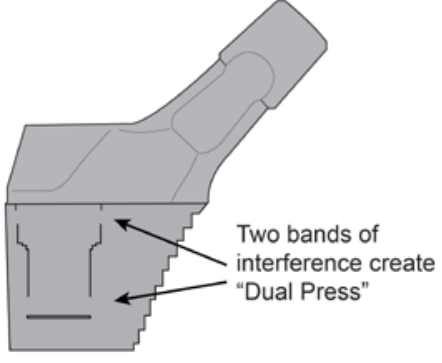

Dual Press ${ }^{\mathrm{TM}}$ Technology

Figure 1. Illustration of Press Fit

gle. The diameter of the locating pin was $3.175 \mathrm{~mm}$ and would engage with one of three holes within the interface of the modular shoulder/neck. This provided $13^{\circ}$ of version control. One of three positions could be picked, $13^{\circ}$ of anteversion, neutral, or $13^{\circ}$ of retroversion. Figure $5 \mathrm{~A} \& \mathrm{~B}$.

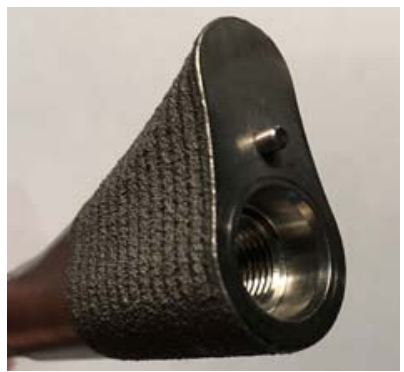

Figure 5A. Picture of stem body showing location / derotational pin. (Courtesy JISRF Archives)

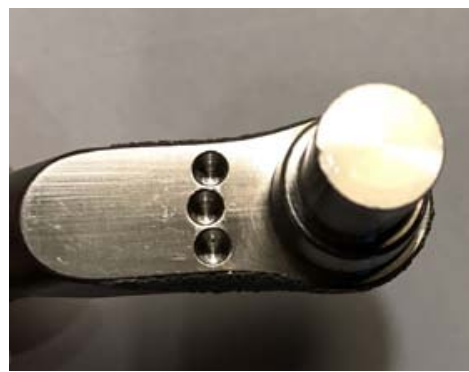

Figure 5B. Underside of neck showing 3 pin holes for version angle. (Courtesy JISRF Archives)

The Apex Modular TM Hip Stem was developed as an evolutionally design based off the successful historical S-Rom ${ }^{\circledR}$ Modular Hip Stem $[\underline{3}, 4]$. The basic stem geometry was similar to the S-Rom in that both were straight stems with a proximal fit and fill cone and a medial triangle shape. Figure $3 \mathrm{~A} \&$ B.

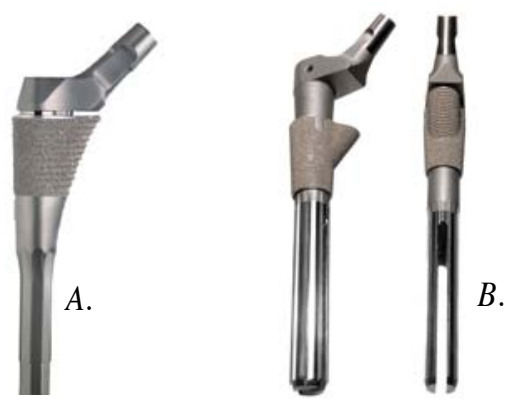

Figures 3A, and B. A: Apex Modular Stem. Note: Proximal modular shoulder/neck not fully seated (Courtesy George Cipolletti). B: S-Rom( Stem (Courtesy JISRF Archives).

Stem features a novel proximal modular shoulder/neck design that is not a taper junction. This modular junction is a Dual Press. This attachment mechanism is new to orthopaedics but was derived from conventional mechanical tool designs. The entire shoulder of the neck sits flush onto the stem body thus shares load over a larger surface area vs. a taper junction. This provides fatigue values equal to that of a monob-

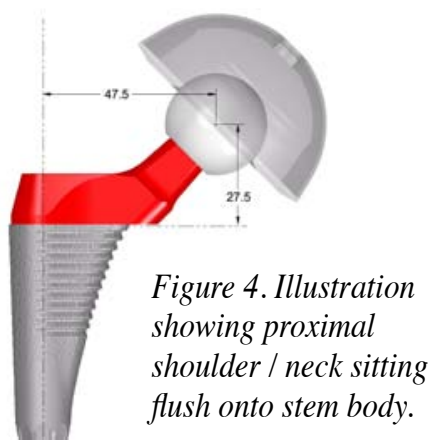
lock stem. Figure 4.

An alignment pin engages with a mating hole on the distal surface of each modular neck. This provides additional torsional stability, as well as control of version an-

The original design included pre-clinical testing of the following worst-case scenario [ㄷ]:

- Six size 2, 9mm stems with medium 42.5 necks, +7 heads (for a total femoral offset of $47.5 \mathrm{~mm}$ ).

- Assembly forces measured for three stems.

- All six stems fatigued tested as per ISO 7206-4 and 7206-8, under the direction of A. Seth Greenwald, D. Phil (Oxon)

- All six stems survived 5 million cycles at $2300 \mathrm{~N}$.

- Same stems loaded to $81.3 \mathrm{~kg}$ of torsion, then axial tension to disassembly, or $1000 \mathrm{lb}$ limit.

- Fretting wear measured.

Additional testing of high cycle fatigue testing was performed []․

- Size 6, $14.5 \mathrm{~mm}$ stem with medium 47.5 neck, +7 head (for a total femoral offset of $52.5 \mathrm{~mm}$ ).

- Fatigue tested as per ISO 7206-4, with incrementally increased cyclic loads.

- Stem survived 48.5 million cycles, test halted due to failure of embedding material. Maximum load reached 6xBW, for an $81.6 \mathrm{~kg}$ individual $(492.6 \mathrm{~kg})$.

- Titanium debris average less than $0.001 \mathrm{~mm} 3$ per million cycles (less than $0.1 \%$ of wear of MOM hips)

\section{Failure Mode for Generation I "Apex Modular Stem”}

The failure of the Apex Modular Junction was primarily limited to the locating/ de-rotational pin. The pin fractured allowing the proximal shoulder/neck piece to rotate back and forth against the proximal portion of the modular stem. This resulted in micro to macro motion, joint instability, pain and excess generation of titanium debris $[\underline{2}, \underline{8}]$. Figure 6.

Patients often heard an initial snapping sound and a 
sense of hip instability, progressing to a painful hip. Lateral X-Ray views would demonstrate the implants to be rotated out of position and on rare occasion totally disengage from the Dual Press modular junction. Revision of the failed implant is not overly difficult. The proximal neck can be removed by hand provid-

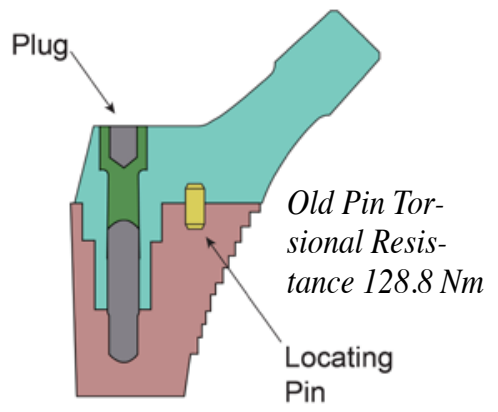

Figure 6. Illustration showing crosssection of Generation I and locating pin that was the point of failure. Pin diameter 3.175 mm. (Courtesy Anna Farad)

ing direct access to the stem body. Retrieval instruments allowed for firm attachment and with the help of flexible ostetomes or a small high-speed burr you can break the bony attachment with minimal bone destruction. Often femoral replacement can be done with a primary length stem. Figures 7A, B, \& C.

Since the two modular pieces are titanium there often would be considerable black debris and staining of the tissues making one think of corrosion. This was not the case. Titanium is a relatively soft material and abrasion debris

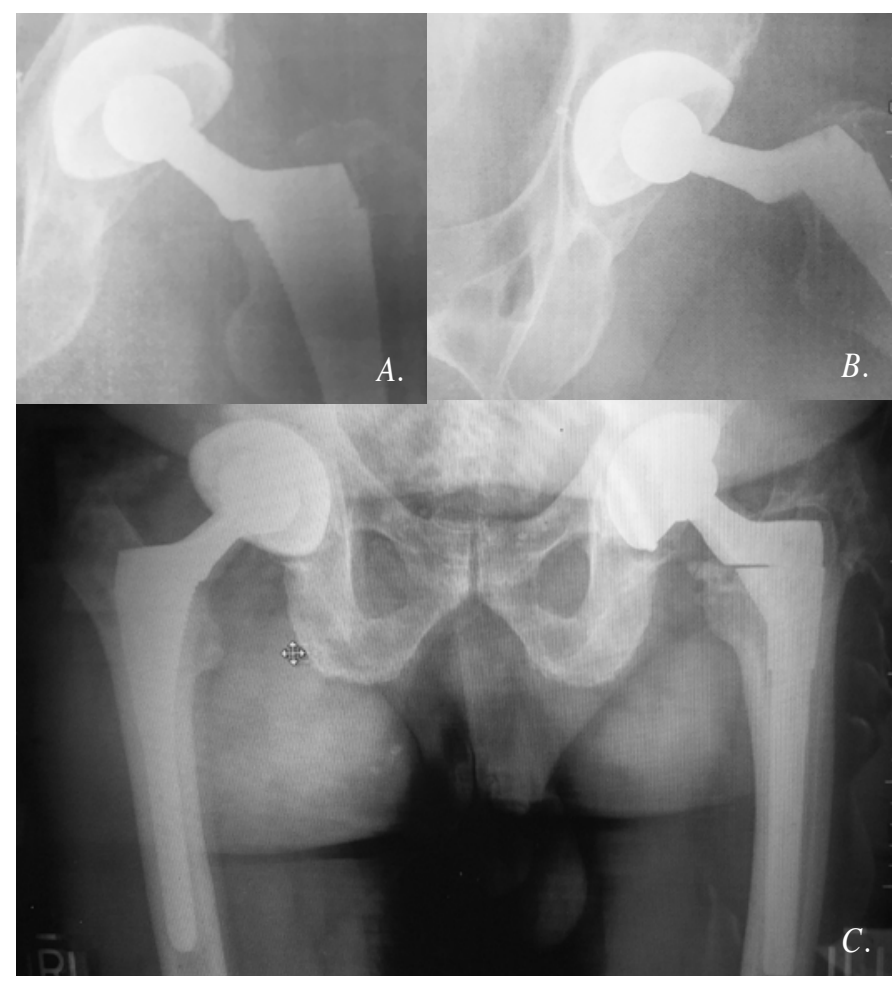

Figures 7A, B, and C. A: Post-op A/P X-ray of a fractured locating/ de-rotational pin. Note: is difficult to appreciate the pin has been broken on this view. B: Lateral X-Ray demonstrating modular should/ neck component has rotated out of position of function. $C$ : A/P X-ray showing bi-lateral hips with Apex Modular stem on the left side, and a S-Rom (primary stem-length) that replaced the fractured locating/ de-rotational pin on the right. (Courtesy T. Tkach) is easily generated. You might also think the fractured location/ de-rotation pin was a fatigue failure problem. This was also not the case. Figures 8, 9A, B, \& C.

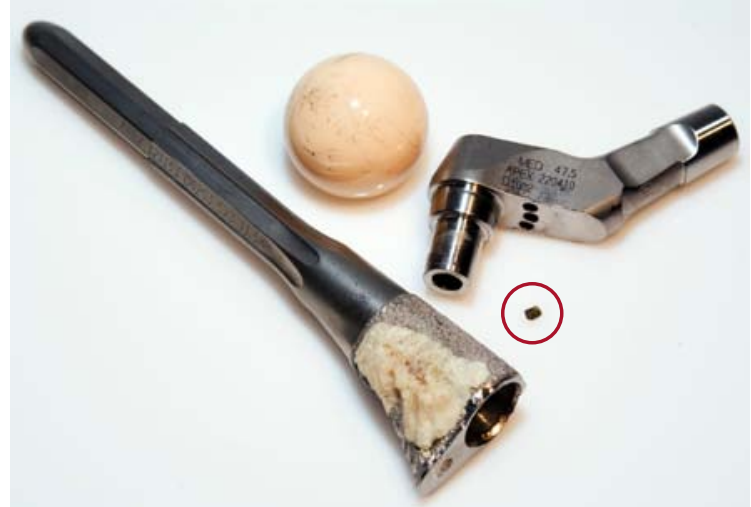

Figure 8. Retrieved Apex Modular Stem showing broken locating pin with no other apparent damage to Dual Press Modular Junction. Note: Good bone attachment to porous surface. (Courtesy JISRF Archives)

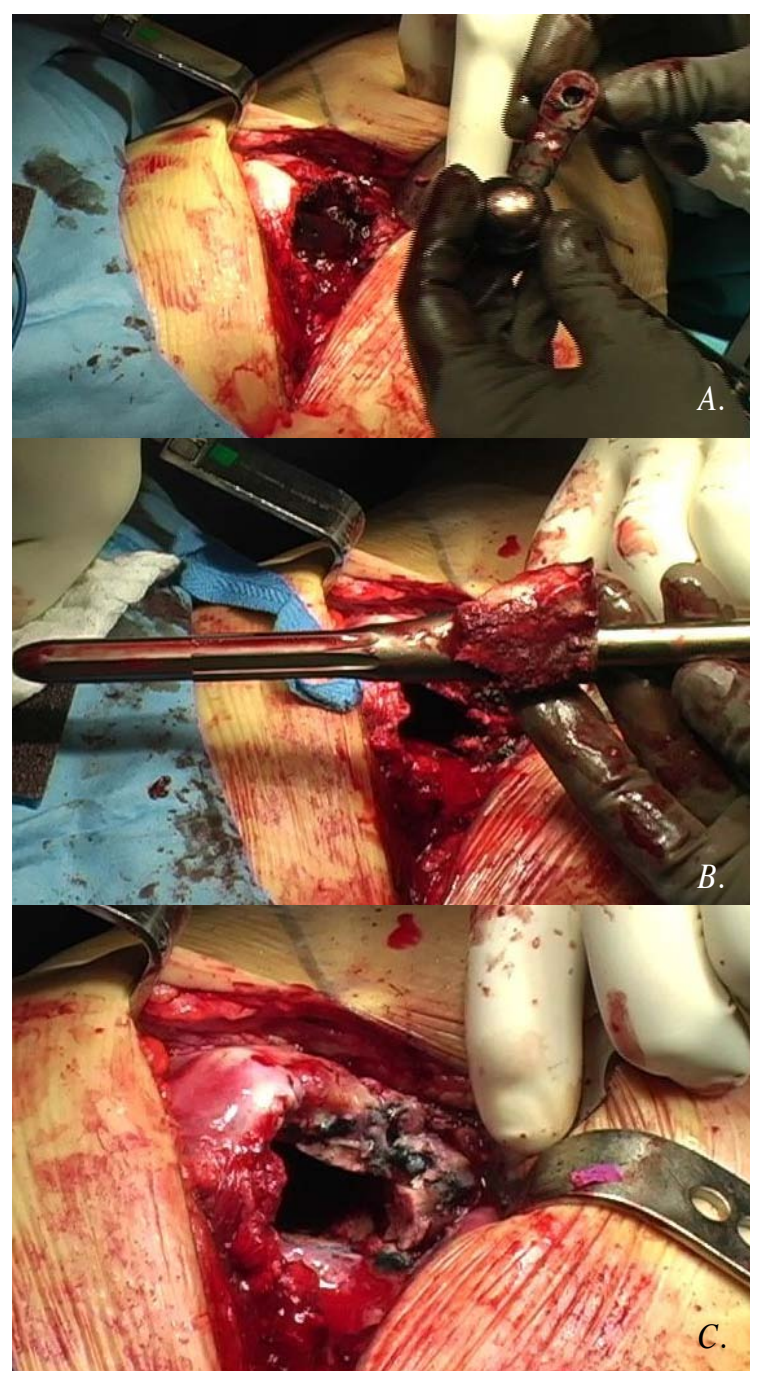

Figures 9A, B, and C. A: Picture showing proximal modular shoulder/neck with femoral head attached. B: Showing stem removed with significant bone attached to proximal porous area with retrieval instrument attached $C$ : Showing stem removed with black titanium debris. Note: this is not corrosion (Courtesy JISRF Archives). 
Metallic fatigue failures clearly demonstrate upon examination a surface fingerprint. There will be a fatigue source or crack initiation then a crack propagation resulting in fatigue failure. Although fatigue has been thought of as a function of time it has been shown that it is the number of repetitions of stress rather than mere duration of time.

So it is important to realize that fatigue cycles are accumulative and this has been the historical failure mode of fractured total hip stems [ㅁ, 10] (Figure 10).

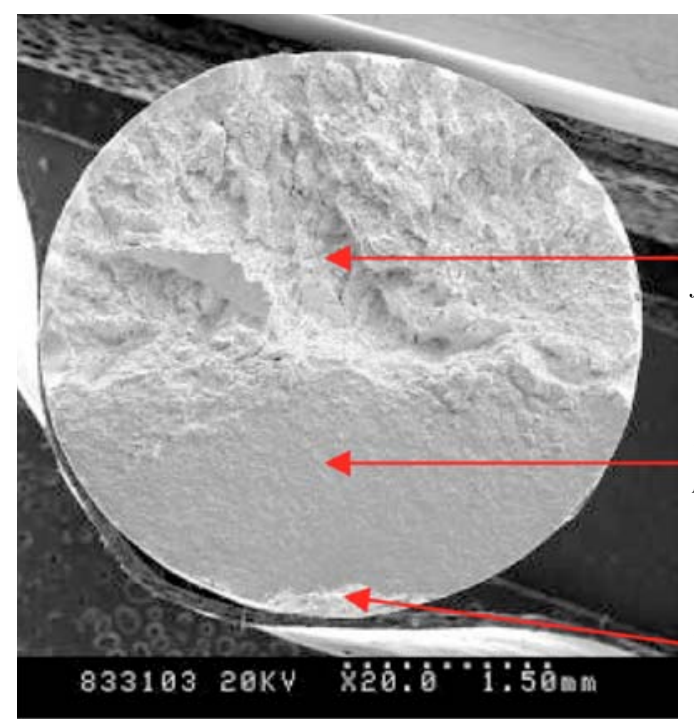

Fatigue

fracture

Fatigue crack propagation

Fatigue source crack initiation

Figure 10. Typical cross-sectional image of a metal fatigued failure showing source, propagation and fracture. (Courtesy JISRF Archives source unknown.)

Upon inspection of the retrieved fractured pins (c.c.) there was no evidence of a fatigue failure [11]. So what was the cause of the fracture? The hypothesis is a dynamic high impact torsional shear failure marked by a vigorous physical force applying a load well beyond the shear strength of the material. Another way of expressing this would be a moment of momentum that produces a load beyond the shear strength of the material. Example jumping off the bed of a pick-up truck landing with your foot internally rotated or possibly stumbling could generate a high dynamic impact torsional load resulting in a shear failure of the locating/de-rotational pin.

It was further thought that the Dual Press plug would have additional property values that would contribute to the overall integrity of the composite design, which in hindsight had little torsional resistance value.

\section{Results for Generation I "Apex Modular Stem”}

Generation I, 483 stems were implanted between 2000 and 2004. 31 alignment pins sheared resulting in a revision rate $6.4 \%$.

The stem system was voluntary withdrawn from the market and redesigned to a more robust design.
Table 1: Generation I Results

\begin{tabular}{|r|r|l|}
\hline \multicolumn{2}{|c|}{ Total Implants from 2000 - 2004 } & 483 \\
\hline Revisions & Pin Shear & 31 \\
\cline { 2 - 3 } & Infection & 2 \\
\cline { 2 - 3 } & Dislocation & 1 \\
\cline { 2 - 3 } & Total & 34 \\
\hline \% Revisions & Pin Shear & $6.4 \%$ \\
\cline { 2 - 3 } & Infection & $0.4 \%$ \\
\cline { 2 - 3 } & Dislocation & $0.2 \%$ \\
\cline { 2 - 3 } & Periprosthetic Fracture & $0.0 \%$ \\
\cline { 2 - 3 } & Total & $7.0 \%$ \\
\hline
\end{tabular}

\section{Generation II Design “Omni Mod Stem”}

The Omni Mod Stem design is the exact same features of the proximal shoulder / neck and the stem body from Gen I with changes to the structures within the Dual Modular junction (plug to bolt and diameter of locating pin from $3.175 \mathrm{~mm}$ to $4.775 \mathrm{~mm}$ ). The dimensions for the two bands of interference fit remained the same.

The increase in pin diameter resulted in creating two different shoulder / neck configurations. One style has a single engagement hole for neutral version and a second style that has two holes for selection of anteversion or retroversion at $13^{\circ}$. (Figure $11 \mathrm{~A}, \mathrm{~B}, \& \mathrm{C}$ )

Figures $11 A, B \&$ C. A: Larger diameter pin (c.c. $4.775 \mathrm{~mm}$ ) within the stem body of the Dual Press Modular junction. B: A single engagement hole that provides a neutral neck position. $C$ : Two holes that provide $13^{\circ}$ of version angle. (anteversion or retroversion)

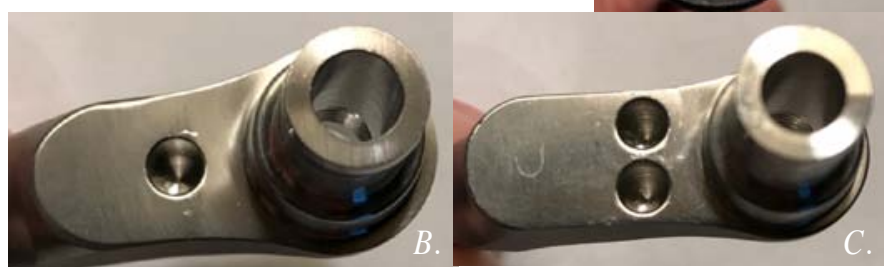

This corrective action resulted in $225 \%$ increase in torsional strength. It serves as an example that changes and improvements are possible once there is a full understanding of the problem. There have been no reported mechanical failures of its modular junction since 2004 with the improved design "Omni MOD Stem" (Figure 12). [13] 
Figure 12. Illustration crosssection of improved Dual Press on Omni Modular Generation II Design increased pin diameter to $3.175 \mathrm{~mm}$ increased torsional strength by 225\%. (Courtesy Anna Farad)

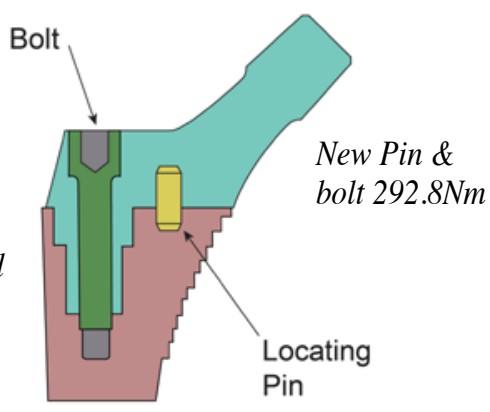

\section{Results for Generation II “Omni Mod Stem”}

1,642 stems have been implanted since

2004 and 2016 utilizing the same stem surgical technique. There have been no pin shear failures since this more robust design has been introduced.

There have been no reported complications with the improved "Dual Press" Modular Junction.

Note: There have been two reported fractured necks from Australia, not the modular junction, as with conventional monoblock stem designs.

\section{Discussion}

The knowledge of implant failure and implant testing is continuing to grow but often as we solve one mode of failure we create another failure that has not been anticipated. Historical review and preclinical testing might meet the required standards set by regulatory bodies to achieve market release, but often these standards do not consider the ever-increasing physical activity and loads that these devices are encountering [10].

It was further thought that the Dual Press plug would have additional property values that would contribute to the overall integrity of the composite design, which in hindsight had little torsional resistance value.

Historical torque levels in our opinion have been underestimated in today's patient life styles that demonstrate increased physical activity. Previous studies have demonstrated torque values ranging between $15 \mathrm{Nm}$ (11 ft-lbs) and $37 \mathrm{Nm}$ (27 ft-lbs) depending on the physical activity (rising from chair to single-limb stance) []].

The trends over the past ten years have been the use of large femoral heads, increased femoral offset, metal on metal bearings along with increased patient activity. All of these factors increase torque []. On average, a 1-mm true lateral increase to the ball center offset will increase torque values by $8 \%$. A 1-mm increase in vertical height (leg length) will increase torque by $6 \%$ []. Torque is a force applied over a distance (lever arm) that causes rotation about a fulcrum (axis of rotation) (Torque=Force

Table 2: Generation II Results
\begin{tabular}{|r|r|l|}
\hline Total Implants from 2004 - 2016 & 1642 \\
\hline Revisions & Pin Shear & 0 \\
\cline { 2 - 3 } & Infection & 4 \\
\cline { 2 - 3 } & Dislocation & 1 \\
\cline { 2 - 3 } & Periprosthetic Fracture & 1 \\
\cline { 2 - 3 } & Total & 6 \\
\hline \% Revisions & Pin Shear & $0.0 \%$ \\
\cline { 2 - 3 } & Infection & $0.2 \%$ \\
\cline { 2 - 3 } & Dislocation & $0.1 \%$ \\
\cline { 2 - 3 } & Periprosthetic Fracture & $0.1 \%$ \\
\cline { 2 - 4 } & Total & $0.4 \%$ \\
\hline
\end{tabular}

(Fm) x Moment Arm). The greater the torque a muscle can produce, the greater the movement it will produce on the body's levers.

We now know by experience that the hip sees torque values over $(128.8 \mathrm{Nm})$, as demonstrated in our mechanical failures of the Apex Modular hip stems [10, 12] (Figure 13).

This paper follows on previous publications of this unique modular junction and demonstrates that design and materials can be improved upon once there is clear understanding of the failure mode. It is important to remember all devices are subject to failure. It is also necessary to recognize design and material limits and not to over-indicate in high-risk patients. Patient activities are higher and generate higher mechanical loads than historical references.

A number of modular junctions have come and gone from clinical use. Nevertheless, the endeavor to improve clinical outcomes should be continued.

Modularity can be designed and fabricated to provide safe, reliable, and reproducible clinical results. Because there are no laboratory tests allowing accurate prediction of the service life and performance of implant parts, clinical experience with a large number of cases over a period of several years is the only reliable indicator. However, clinical evaluations should only begin after conducting aggressive basic science material and mechanical testing to anticipate potential failure modes. Individual patient physical activities should be considered when deciding on stem modularity features. Since there are no standards established for modular junctions the overall performance of modular junctions are not equal. Careful review of basic engineering principles is necessary and recognizing design limits will reduce the indication of overuse $[\underline{2}, \underline{4}, \underline{8}, \underline{9}, \underline{10}]$.

We encourage early publication of all devices (good, bad \& ugly) and continuation of those publications as clinical experience and outcomes become available. 


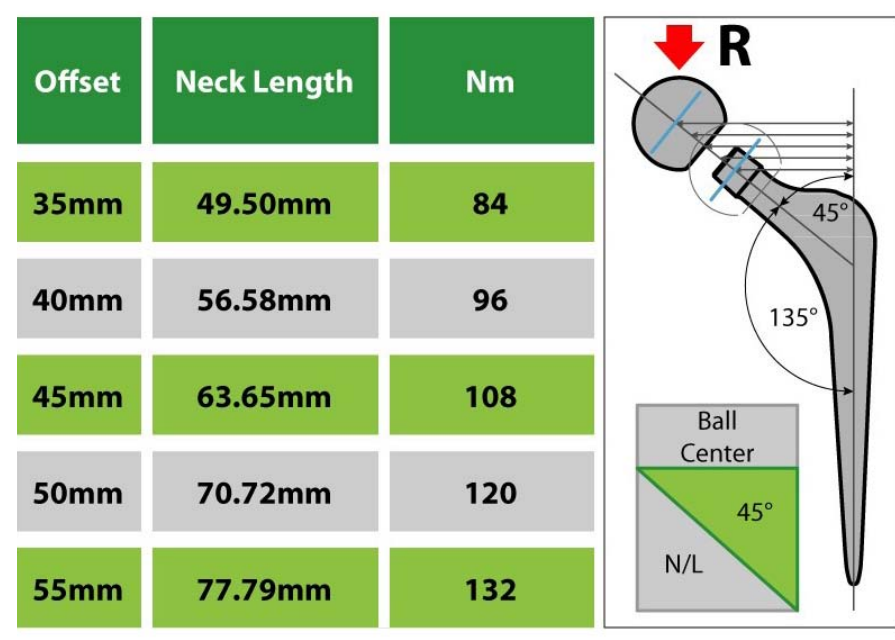

Figure 13. Chart Showing Torque Loads Generated by Femoral Offset and Neck Length

\section{Conclusion}

All implant devices entail a multitude of risks and benefits. The Apex Modular Stem (Generation I), provided excellent fixation, minimal risk of modular junction corrosion, and simple control of anteversion and femoral offset. The limitation was found to be the risk of the alignment pin shearing (6.4\%). The pin was enlarged to make it $225 \%$ stronger in torsional resistance, and in a subsequent series of over 1,600 femoral stems in a single surgeon series, there were no pin failures over a 12 year duration.

Improvements in this modular junction design have eliminated the mechanical failures of the first Generation design.

\section{References:}

1. Cameron H.U. The Technique of Total Hip Arthroplasty. ISBN 0-8016-0825-2 1992 by Mosby-Year Book.

2. Tkach T, Low W, Cipolletti G.B., McTighe T. Target Restoration of Hip Mechanics in THA. AAOS Scientific Exhibit 2006, DOI: 10.13140/RG.2.1.1084.0167

3. Cameron H.U., Yung-Bok, J., Noiles, D.G., McTighe, T. Design Features and Early Clinical Results with a Modular Proximally Fixed Low Bending Stiffness Uncemented Total Hip Replacement. DOI: 10.13140/RG.2.1.4714.7040 Conference: AAOS 1988 Annual Meeting, Scientific Exhibit
4. Keppler, L., McTighe, T., The Role of Stem Modularity for THA in a Community Based Practice. Reconstructive Review Volume 2(Number 2): 16 · August 2012 DOI: $10.15438 /$ rr.v2i2.10

5. Sales Training Slide Presentation by Omni (On file)

6. G. M. Kotzar, D. T. Davy, J. Berilla, and V. M. Goldberg. Torsional Loads in the Early Postoperative Period Following Total Hip Replacement. JBJS 13:945-955

7. T. W. Phillips, * L. T. Nguyen and S. D. Munro. Loosening of Cementless Femoral Stems: A Biomechanical Analysis of Immediate Fixation with Loading Vertical, Femur Horizontal. J. Biomechanics Vol. 24, No 1, pp. 37 48, 1991. 9,10

8. Keggii K, Keggi J, Kennon R, Tkach T, Low W, et al., Within Any Important Issue, There are Always Aspects No One Wishes to Discuss- Femoral Component Failure. Poster Exhibit ISTA, Oct. 2006

9. Jameel A, Majeed W, Razzaq A, Fatigue Analysis of Hip Prosthesis. Number 10, Vol 18, October 2012 Journal of Engineering p: 1100 to 1114

10. Mierzejewska Z, Case Study and Failure Analysis of a Total Hip Stem Fracture Advances In Materials Science, Vol. 15, No. 2 (44), June 2015 DOI: $\underline{10.1515 /}$ adms-2015-0007

11. T. McTighe, D. Brazil, W. Bruce. Metallic Alloys in Total Hip Arthroplasty. McTighe T, Brazil D, Bruce W. Metallic Alloys in Total Hip Arthroplasty. In: Cashman J, Goyal N, Parvizi J, eds. The Hip: Preservation, Replacement and Revision. Baltimore, MD: Data Trace Publishing Company; 2015:14-1-14-12

12. McTighe T, Brazil D, Clarke I, Keppler L, Keggi J, Tkach T, McPherson E. Metallic Modular Taper Junctions in Total Hip Arthroplasty. RR Vol 5, Number 2, July 2015 DOI: $\underline{10.15438 / \mathrm{rr} .5 .2 .108}$

13. White paper published by Omni 2004

\section{SUBMISSION HISTORY}

Submitted August 30, 2017

Reviewed September 16, 2017

Revised September 24, 2017

Accepted September 27, 2017

Published September 30, 2017

\section{AUTHOR AFFILIATIONS}

1 Thomas Tkach, MD • McBride Orthopedic Hospital, 1110 N Lee Ave, Oklahoma City, OK 73103

2 Timothy McTighe, Dr HS (hc) • Joint Implant Surgery \& Research Foundation, 46 Chagrin Shopping Plaza, \#117, Chagrin Falls, OH 44022 US

(Direct inquires to Timothy McTighe, tmct@jisrf.org)

AUTHOR DISCLOSURES

Tkach royalty and equity (Omni); McTighe equity (Omni)

COPYRIGHT \& OPEN ACCESS

(C) 2017 Tkach, McTighe. All rights reserved.

Authors retain copyright and grant the journal right of first publication with the work. Reconstructive Review is an open access publication and follows the Creative Commons Attributionand share them with others for non-commercial purposes as long as they credit the senior author, Reconstructive Review, and the Joint Implant Surgery \& Research Foundation (JISRF). An example credit would be: "Courtesy of (senior author's name), Reconstructive Review, JISRF, Chagrin Falls, Ohio". 